\title{
Die Stickstoffverteilung in der Frauenmilch.
}

\author{
Von
}

A. Frehn.

(Aus der akademischen Klinik für Kinderheilkunde in Düsseldorf, Direktor Prof. Dr. Schlossmann.)

(Der Redaktion zugegangen am 12. Februar 1910.)

Eingehendere Untersuchungen über die Stickstoffverteilung in der Frauenmilch konnten erst vorgenommen werden, nachdem für die Ausfällung des Caseins eine Methode gefunden worden war, die bei leichter Anwendbarkeit eine quantitative Ausfällung des Caseins ohne gleichzeitige Abscheidung anderer Eiweißkörper gewährleistete. Diesen Anforderungen zu genügen, war man lange Zeit hindurch vergebens bemüht.

Die Versuche, das Casein der Frauenmilch, dessen schwere Gerinnbarkeit schon den älteren Autoren bekannt war, mittels der für die Kuhmilch angewandten Methoden quantitativ auszufällen, waren erfolglos.

Anhaltspunkte für die Abscheidung des Caseins aus der Milch waren gegeben, nachdem neben dem Casein noch zwei phosphorfreie Eiweißkörper von kleinerem Molekül in der Milch mit Sicherheit nachgewiesen waren.

Auf dem Größenunterschiede zwischen Caseinmolekül und dem Molekül der anderen Eiweißkörper fußend versuchte Lehmann ${ }^{1}$ ) eine Reindarstellung des Caseins der Kuh- und Frauenmilch auf mechanischem Wege. Er filtrierte die Milch durch poröse Tonseparatoren und behielt auf dem Filter das Caseinfettgerinnsel, wăhrend die löslichen Eiweißstoffe mit dem Serum das Filter passierten. Das Casein wurde dann nach Entfernung des Fettes gewichtsanalytisch bestimmt. Die auf diese Weise gefundenen Caseinwerte betrugen für Frauenmilch 1,2\% (für Kuhmilch 3,0\%).

Die Methode wurde von Schlossmann ${ }^{2}$ ) etwas modifiziert, indem er statt der Tonseparatoren ballonförmige Filter aus Pukallmasse anwandte, durch welche die Milch unter hohem Drucke hindurchgepreßt wurde. Die Analyse des Filterrückstandes ergab einen Casein-N von 0,098 g

1) Lehmann, Pflügers Arch., Bd. LVI, S. 558.

2) Schlossmann, Diese Zeitschrift, Bd. XXII, S. 197. 
bei einem Gesamt- $\mathrm{N}$ von $0,24 \mathrm{~g}$ in $100 \mathrm{ccm}$ Milch: mit anderen Worten, es betrug der Casein-N $41 \%$ des G. N. ${ }^{1}$ )

Trotz der abfälligen Kritik, die Camerer und Söldner ${ }^{2}$ ) an der Möglichkeit einer mechanischen Trennung der Eiweißkörper der Milch üben, scheinen mir die nach der Schlossmannschen Methode gewonnenen Resultate gut zu sein, da sie, wie sich später zeigen wird, ziemlich gut mit den von mir gefundenen Durchschnittswerten übereinstimmen, während allerdings die Lehmannschen Zahlen sich nicht unbeträchtlich nach oben hin von den meinigen entfernen, was auf die Methodik Gewichtsanalyse - zurückzuführen sein wird.

Etwas näher eingehen möchte ich auf die ebenfalls von Schloss mann (l. c.) für verschiedene Tiermilchen und die Frauenmilch angegebene Kalialaunfällung, da dies Verfahren relativ einfach erscheint und in der Literatur über hiermit gewonnene Resultate an zwei Stellen berichtet wird. Camerer und Söldner (l. c.) heben hervor, daß nur wenige der von ihnen verwandten Frauenmilchen bei vorsichtigem Zusatz von Kalialaun überhaupt einen Niederschlag gaben. Derselbe sei vielmehr meist erst nach NaCl-Zusatz eingetreten, aber auch selbst da oft ausgeblieben. Filtrierbar seien die Proben nach Zusatz von Calciumphosphat meist gewesen. Die Caseinstickstoffwerte, welche Camerer und Söldner bei einer Reihe von Untersuchungen nach dieser Methode gefunden haben, schwanken zwischen 39,6 und $69,39 \%$ des G. N.

Zaits check, 9) der sich zum Studium der Pepsinsalzsäurelöslichkeit des Caseins der Frauenmilch Casein nach der Schlossmannschen Methode herstellte, gibt 34,6, 38,7 und $40 \%$ des G. $N$ als Casein-N an. Nähere Angaben über die Fällungseigentümlichkeiten sind an dieser Stelle nicht $\mathrm{zu}$ finden.

Ich selbst habe, um Vergleichswerte mit anderen Methoden zu erhalten, die Alaunfallung ebenfalls versucht, das eine Mal mit, das andere Mal ohne NaCl-Zusatz. Bei der ersten Probe war eine Fällung trotz Zusatz mehrerer Kubikzentimeter gesättigter Alaunlösung kaum zu konstatieren, bei der zweiten mit $\mathrm{NaCl}$ versetzten Probe war nach Zusatz mehrerer Kubikzentimeter Alaun und mehrstündigem Stehenlassen im Wasserbade von $40^{\circ}$ eine Ausfallung zwar vorhanden, ein klares Filtrat jedoch trotz Zusatzes von Calciumphosphat und mehrmaligen Filtrierens nicht $\mathrm{zu}$ erhalten.

Das Manko dieser Fällungsmethode scheint mir, soweit man überhaupt aus zwei Versuchen einen Schluß ziehen darf, darin zu liegen, daß der Eintritt der Gerinnung wegen der außerordentlich feinflockigen Aus-

1) In der ganzen Arbeit ist zu lesen: G. $N=$ Gesamtstickstoff; F. $\mathrm{N}=$ Filtratstickstoff; C. $\mathrm{N}=$ Caseinstickstoff ; R. $\mathrm{N}=$ Reststickstoff.

$\left.{ }^{2}\right)$ Camerer und Söldner, Z. f. Biol., Bd. XVIII, S. 277.

$\left.{ }^{3}\right)$ Zaitscheck, Pflügers Arch., Bd. GIV, S. 550. 
fällung nur schwer erkannt werden und daher eine Dosierung des Fällungsmittels nur unsicher erfolgen kann. Es besteht infolgedessen die Möglichkeit, daß neben dem Casein noch andere Eiweißkörper mit ausgefällt werden. So werden vielleicht auch die von Camerer und Söldner gefundenen hohen Caseinwerte (bis $69 \%$ des G. N) verständlich.

Die Ursachen für die Schwierigkeit, Frauenmilchcasein quantitativ auszufällen, hat man in verschiedenen Richtungen gesucht. Man dachte an die starke Verdünnung, in welcher sich das Casein der Frauenmilch im Vergleich $\mathrm{zu}$ dem der Kuhmilch befindet, man beschuldigte seinen hohen Albumingehalt, durch den die für die Gerinnung erforderliche Annäherung der Moleküle im Raume verhindert werde, man hob die biologischen Eigentümlichkeiten des Frauencaseins hervor, durch die eine Differenzierung der Frauenmilch von der Kuhmilch ermöglicht wird, man dachte auch an die auf elementar-analytischem Wege gefundenen chemischen Unterschiede zwischen beiden Caseinen und hat neuerdings in dem Opalisin (Wroblewski ${ }^{1}$ )) denjenigen Eiweißstoff zu erkennen geglaubt, welcher die Ausfällung des Caseins erschwere. Einen Einblick in das eigentümliche Verhalten des Frauencaseins den verschiedenen Fällungsmethoden gegenüber vermögen diese Erklärungsversuche jedoch nicht zu geben.

Das Verdienst, die erste brauchbare Methode der Caseinfällung in der Frauenmilch geschaffen zu haben, gebührt ohne Frage Kobrack. ${ }^{2}$ )

Aus dem differenten, bereits längere Zeit bekannten Verhalten der Kuh- und Frauenmilch bei Säurezusatz, nämlich, daß das Kuhcasein aus den Lösungen seiner Salze in dem Augenblick quantitativ ausfällt, wo die Lösung rotes Lackmuspapier nicht mehr bläut, während das Frauencasein bei gleicher Behandlung seiner Lösung absolut unverändert bleibt, zog Kobrack den Schluß, daß in der Frauenmilch irgend welche Stoffe vorhanden sein müßten, welche die Gerinnung des Caseins hinderten. Um diese zu entfernen, dialysierte er die angesäuerte Milch und zwar gegen Chloroformwasser, weil er der Ansicht war, daß auch die nach dem Zentrifugieren in der Milch noch vorhandenen Fettpartikelchen der Gerinnung hinderlich sein könnten. Die Fällung der Frauenmilch durch Essigsäure gelang auf diese Weise in der Tat. Neuerdings wurden nun erhebliche methodische Fortschritte gemacht.

Fuld und Wohlgemuth ${ }^{3}$ ) ließsen die Frauenmilch, um ihr Casein von dem ihm eigenen für die Fällung ungünstigen Zustande $z u$ befreien, $3 \times 24$ Stunden in gefrorenem Zustande. Die aufgetauchte Milch hatte alsdann die Fähigkeit bekommen, auf Zusatz geringer Säuremengen (1 Tropfen einer $5 \%$ igen Essigsäure) prompt und leicht zu gerinnen.

1) Wroblewski, Diese Zeitschrift, Bd. XXVI, S. 308.

2) Kobrack, Pfügers Arch., Bd. LXXX, S. 69.

3) Fuld und Wohlgemut, Bioch. Zeitschr., Bd. V, S. 118. 
Dje Wirkung des Einfrierens auf das Caseinmolekül wird von den Autoren so erklärt, daß durch dasselbe eine Beeinflussung des kolloidalen $\mathrm{Zu}$ standes des Caseins im Sinne einer Kornvergrößerung stattfinde.

Eine andere Methode, das Casein der Frauenmilch leicht auszufällen, stammt von B. Bienenfeld ; ${ }^{1}$ ) auf diese werde ich weiter unten ausführlicher zurückkommen.

Die neueste, außerordentlich leicht und sicher auszuführende und allen Anforderungen einer quantitativen Reindarstellung des Caseins genügende Methode stammt von Engel. $\left.\left.{ }^{2}\right)^{3}\right)^{4}$ ) Sie bildet die Basis meiner Untersuchungen und wurde, um es noch einmal kurz mitzuteilen (die Methode ist in der Bioch. Zeitschr. 08, Bd. XIII genauer beschrieben), so ausgeführt, daß zu $40 \mathrm{ccm}$ frischer Milch, die gewöhnlich zur Bestimmung verwendet wurden, etwa $15-30 \mathrm{ccm}$ einer n/10-Essigsäure gesetzt wurde. Die Milch erhielt also ungefähr eine Acidität von 4-8 ccm n/10-Essigsäure auf $10 \mathrm{ccm}$. Das Gemisch wurde, da eine 4-5malige Verdünnung sich für die Ausfällung als sehr zweckmäßig erwies, auf 200 aufgefüllt und mehrere Stunden (3 Stunden sind ausreichend) in den Eisschrank gesetzt, doch ohne es einfrieren zu lassen, und alsdann der Einwirkung eines Wasserbades von $40^{\circ}$ ausgesetzt. Die Essigsäure wurde darum bevorzugt, weil bei ihr das Optimum der für die Fällung nötigen Acidität bei weitem nicht so eng umgrenzt ist, wie bei anderen Säuren, es pflegt vielmehr bei einer Acidität von $4-12 \mathrm{ccm}$ n/10-Essigsäure auf $10 \mathrm{ccm}$ Milch wie Engel sich ausdrückt, « ein breites Plateau optimaler Ausflockung erreicht zu werden ». Das hat den großen Vorteil, daß man die Methode in ihrer vorhin angegebenen Form ohne Bedenken auf die verschiedenen Milchsorten anwenden kann, ohne jedesmal die optimale Acidităt von neuem zu ermitteln. Sobald nun die Milch im Wasserbade sich $z u$ erwärmen beginnt, tritt auch in den meisten Fällen bereits die Ausflockung ein. Eine ganz offensichtliche Unterstützung - darauf hat ebenfalls Engel (l. c.) schon hin-

1) Bienenfeld, Biochem. Zeitschr., Bd. VII, S. 262.

2) Engel, ibid., Bd. XIII, S. 89.

s) Engel, ibid., Bd. XIV, S. 234.

4) Engel, Die sFrauenmilchs in Sommerfelds aHandbuch der Milchkundes, Wiesbaden 1909 bei J. F. Bergmann. 
gewiesen - erfährt die Gerinnung entgegen den Beobachtungen anderer Autoren, deren Methodik allerdings eine andere war, durch die Anwesenheit des Fettes in der Milch. Daher wurde $\mathrm{zu}$ den Analysen stets unzentrifugierte Milch genommen.

Die Rolle, welche die Fetttröpfchen bei der Ausfällung des Caseins spielen, kann vielleicht mit dem begünstigenden Einfluß, den feste Körper für das Auskrystallisieren von Salzen aus ihren Lösungen einnehmen, in Parallele gesetzt werden, oder aber sie sind nur indirekt und zwar rein mechanisch bei dem Gerinnungsvorgange beteiligt, indem sie die Bildung von größeren Komplexen begünstigen. Die Fällung des Caseins ist als beendet anzusehen, sobald eine einigermaßen deutliche Scheidung von dem auf der Flüssigkeit schwimmenden Caseinfettgerinnsel und der Molke eingetreten ist. Die Filtration bereitet keine größeren Schwierigkeiten und ist schnell vollendet. Die ganze Handhabung der Methode ist daher die denkbar einfachste.

Wichtiger als dies ist die Frage:

Wo sind die Garantien, die diese Methode für eine quantitative Fällung des Frauenmilchcaseins liefert? Sie ergeben sich ungezwungen aus folgendem:

Bei allen Versuchen wurde mit Genauigkeit darauf geachtet, ein wasserklares Filtrat zu erhalten. Das ließ sich bei den meisten Milchen schon nach 2--3maligem Zurückgießen der ersten Kubikzentimeter des Filtrates unschwer erreichen. Einige Milchen waren sogar schon nach 1 maliger Filtration absolut klar. Andere der untersuchten Milchen jedoch bildeten eine Ausnahme von dieser Regel insofern, als die Filtration infolge großer Feinflockigkeit der Gerinnsel öfter wiederholt werden mußte, und vereinzelte wiesen sogar nach fortgesetzter Filtration nicht jene tadellose Klarheit auf, wie sie gewöhnlich $\mathrm{zu}$ finden war, sondern zeigten einen Schimmer von Opalescenz. Die nächstliegende Erklärung dafür ist; daß in dem Filtrate noch Spuren unveränderten Caseins vorhanden sind. Mit dem Fettgehalte der Milch hat die Erscheinung offenbar nichts zu tun, da sie bei fettarmen und fettreichen Milchen $\mathrm{zu}$ beobachten ist. Doch scheint die Menge der zugesetzten Essigsäure dabei 
eine Rolle zu spielen, wie aus einem bei derselben Milch angestellten Parallelversuche hervorgeht, bei dem nach Zusatz der gewöhnlichen Säuremenge ein absolut klares, nach Zusatz der doppélten Menge ein leicht opalescierendes Filtrat erhalten wurde. Man wird sich eben zweckmäßig nicht gerade an die Grenzen der für die Fällung möglichen Acidität begeben. $\mathrm{Zu}-$ dem kann es sich bei leicht getrübter Molke nur um Spuren nicht gefällten Caseins handeln, denn die N-Werte waren bei beiden Parallelbestimmungen genau die gleichen. Die Mengen Casein, wie sie dem Auge noch eben als leichte Opalescenz wahrnehmbar sind, sind eben so winzig, daß sie auf chemischem Wege, durch Bestimmung des N-Gehalts, nur bei Anwendung größerer Flüssigkeitsvolumina noch nachweisbar sind. Das ergibt sich auch leicht aus folgendem Experiment: Verdünnt man scharf zentrifugierte Frauenmilch, deren eigentümliche blauweiße Farbe in der Hauptsache auf das Casein zurückzuführen ist, mit Wasser, so kann man bei einer Dicke der Flüssigkeitsschicht von etwa $8 \mathrm{~cm}$ eine Milchverdünnung von ungefähr 1/5000 deutlich vom klaren Wasser unterscheiden. Den Caseingehalt der Frauenmilch zu 0,5\% angenommen (s. h.) würden $500 \mathrm{ccm}$ der Verdünnung $0,0005 \mathrm{~g}$ Casein enthalten. Mit anderen Worten besagt dies, daß eine leicht opalescierende Molke in der Menge von $500 \mathrm{ccm}$, das würde $100 \mathrm{ccm}$ Milch entsprechen, nur ungefähr 0,0005 Casein enthält, eine Menge, die man ohne Bedenken unberücksichtigt lassen kann.

Einen weiteren Beweis für die Zuverlässigkeit der angewandten Methode bildet die Übereinstimmung ihrer Resultate mit denen einer anderen, ebenfalls auf Säurefällung gegründeten Methode, ich meine die Milchsäurefällung nach B. Bienenfeld (1. c.). B. Bienenfeld ging bei ihren Versuchen so vor, $\mathrm{da} B$ sie die Acidität der frischen, zentrifugierten Frauenmilch durch Zusatz bestimmter Mengen n/10-Milchsäure auf 2,8-3,0 Gesamtacidität steigerte, auf das $4-5$ fache mit Wasser verdünnte und das Gemisch solange in ein Wasserbad von $40^{\circ}$ stellte, bis eine deutliche Ausflockung vorhanden war. Die. Filtration soll stets ein klares Filtrat ergeben haben. Die so gefundenen Caseinwerte schwanken zwischen $16-20 \%$ des 
G. N. In 2 Fällen, in denen etwas ältere Frauenmilch ${ }^{1}$ ) mit einer Acidität von 13 zur Verwendung kam, war der gefundene Caseinstickstoffgehalt ein höherer und betrug 52,6\% des G. N. Es wurde nun von mir bei verschiedenen frischen Mischmilchen der Caseingehalt nebeneinander nach Engel und Bienenfeld bestimmt. Die so erhaltenen Resultate enthält nachstehende Tabelle I. Man sieht aus der Tabelle nicht allein, $\mathrm{da} \beta$ in keinem Falle trotz geringer Acidität der untersuchten Milchen jene niedrigen Caseinwerte gefunden wurden, wie sie Bienenfeld selbst nach ihrer Methode analysiert haben will, sondern auch, daß eine auffallende Übereinstimmung zwischen den nach beiden Methoden gefundenen Caseinwerten besteht. Daraus folgt, daß es sich wohl beide Male um die Abscheidung eines in seiner Menge feststehenden Körpers gehandelt hat. Anders wäre die große Übereinstimmung der Resultate nicht gut zu erklären. Gleichzeitig läßt die Tabelle erkennen, daß die niedrigen von $B$. Bienenfeld selbst gefundenen Caseinwerte kaum richtig sein können.

Tabelle I.

\begin{tabular}{c|c|c|c|c|c|c}
\hline \hline & G. N & $\begin{array}{c}\text { F. N in g } \\
\text { nach }\end{array}$ & $\begin{array}{c}\text { C. Nin \% des G. N } \\
\text { nach }\end{array}$ & $\begin{array}{c}\text { Acidität } \\
\text { der Milch } \\
\text { n/10-NaOH }\end{array}$ \\
\cline { 2 - 6 } & pr. 10 ccm \\
\hline $\begin{array}{c}\text { Mischmilch } \\
\text { (zentrifugiert) }\end{array}$ & 0,193 & 0,100 & 0,098 & 48,2 & 49,2 & 0,5 \\
$\begin{array}{c}\text { Mischmilch } \\
\text { feld }\end{array}$ & 0,136 & 0,077 & 0,078 & 43,4 & 42,6 & 0,35 \\
$\begin{array}{c}\text { Mentrifugiert) } \\
\text { Mischmilch }\end{array}$ & 0,118 & 0,078 & 0,078 & 33,3 & 33,3 & 0,8 \\
(nicht zentrifug.)
\end{tabular}

Endlich spricht auch folgender Versuch zugunsten einer quantitativen Fällung des Caseins durch die Essigsäuremethode. Aus $300 \mathrm{ccm}$ Frauenmilch wurde das Casein gefällt; zu dem klaren Filtrate wurde Alménsche Lösung solange hinzugesetzt, bis keine Fällung mehr erfolgte; der Niederschlag, welcher sämtliche Eiweißkörper des Filtrates enthielt, wurde auf Phos-

1) Allerdings auch bei gleichzeitiger Verwendung von Lab. 
phor untersucht. Die Probe fiel zwar positiv aus, die Menge des Phosphors war jedoch so gering, daß sie quantitativ nicht mehr nachgewiesen werden konnte. Das aber hätte der Fall sein müssen, wenn das Filtrat größere Mengen Casein enthalten hätte.

Daß durch die verwendete Essigsäure gleichzeitig mit dem Casein noch andere Eiweißkörper ausgefällt werden, kann wohl als ziemlich ausgeschlossen betrachtet werden. Zwar werden sich Acidalbumine bilden, doch sind diese in verdünnten Säuren löslich und werden unbehindert das Filter passieren.

Eine Hauptaufgabe meiner Arbeit war, auf Grund der Engelschen Fällungsmethode den Caseingehalt der Frauenmilch, über den, wie bereits hervorgehoben, in der Literatur entweder unrichtige, oder doch ganz unzulängliche Mitteilungen vorhanden sind, an Hand eines größeren Materiales festzustellen. Zur Untersuchung diente die Milch der Ammen der Düsseldorfer Kinderklinik und zwar wurden, um von jeder Amme eine möglichst gute Tagesmischmilch zu erhalten, aus beiden Brüsten vor und nach jedem Stillgeschäft gleiche Mengen Milch entnommen. Die Menge des gefällten Caseins wurde in Milligramm $\mathrm{N}$ ausgedrückt. Dieser wurde nach Kjeldahl bestimmt, doch nicht direkt, sondern indirekt durch Abzug des analysierten $F$. N vom G. $N$ unter Vernachlässigung der nach Ausscheidung des Caseinfettgerinnsels eintretenden Volumverminderung der Flüssigkeit, welche sich in jedem einzelnen Falle wohl schwerlich ermitteln ließe. Infolge dieser Art der Berechnung werden die analysierten $\mathrm{C}$. N-Werte nicht absolut genau sein, doch sind die Fehler so außerordentlich gering, daß man sie ruhig vernachlässigen kann.

Das Ergebnis der angestellten Untersuchungen ist in Tabelle 2 niedergelegt. Der C. $\mathrm{N}$ der 27 untersuchten Frauenmilchen bewegt sich $z$ wischen 30,0 und $53,6 \%$ des G. $\mathrm{N}$ und beträgt im Mittel 42,93\%. Rechnet man die $\mathrm{N}-\mathrm{W}$ erte auf Fiweiß (Casein) um, indem man sie mit 6,23 multipliziert, so ergibt sich, daß auf $100 \mathrm{~g}$ der untersuchten Milchen $0,4-0,7 \mathrm{~g}$, im Mittel meist $0,5-0,6 \mathrm{~g}$ Casein kommen. 
Tabelle II.

\begin{tabular}{|c|c|c|c|c|c|c|c|}
\hline \multirow{2}{*}{ Name } & \multirow{2}{*}{$\begin{array}{l}\text { Tage } \\
\text { p.p. }\end{array}$} & \multirow{2}{*}{$\begin{array}{l}\text { Milch- } \\
\text { menge }\end{array}$} & \multirow{2}{*}{ G. $N$} & \multirow{2}{*}{ F. N } & \multicolumn{2}{|c|}{$\begin{array}{c}\text { G. } \mathrm{N}-\mathrm{F} . \mathrm{N} \\
=\mathrm{G} . \mathrm{N}\end{array}$} & \multirow{2}{*}{ Bes. Bemerkungen } \\
\hline & & & & & in $\mathbf{g}$ & $\mid$\begin{tabular}{l|} 
in $\%$ \\
d. G. N
\end{tabular} & \\
\hline Gr. & 11 & 665 & 0,243 & 0,148 & $0,09 \check{5}$ & 39,1 & \\
\hline Ka. & 28 & 1305 & 0,256 & 0,140 & 0,116 & 45,24 & \\
\hline$\gg$ & 30 & 1425 & 0,251 & 0,136 & 0,115 & 45,7 & \\
\hline Kei. & 37 & 1115 & 0,226 & 0,158 & 0,068 & 30,0 & Filtrat leicht opalescierend. \\
\hline Ha. & 38 & 1780 & 0,188 & 0,108 & 0,080 & 42,0 & $\begin{array}{l}\text { Filtration sehr leicht, Filtratt } \\
\text { absolut klar. }\end{array}$ \\
\hline$\triangleright$ & 39 & 1700 & 0,216 & 0,100 & 0,116 & 53,6 & do. \\
\hline Ker. & 40 & 1170 & 0,280 & 0,163 & 0,117 & 40,1 & \\
\hline Be. & 60 & 1420 & 0,203 & 0,112 & 0,091 & 44,5 & \\
\hline$\gg$ & 61 & 1220 & 0,206 & 0,107 & 0,099 & 47,8 & \\
\hline Ha. & 64 & 1660 & 0,192 & 0,118 & 0,074 & 38,4 & $\begin{array}{l}\text { Sehr feinflockige Ausfällung, } \\
\text { Filtrat leicht opalescierend. }\end{array}$ \\
\hline$\triangleright$ & 67 & 1660 & 0,201 & 0,105 & 0,096 & 47,8 & do. \\
\hline Ker. & 67 & 1375 & 0,202 & 0,123 & 0,079 & 39,0 & \\
\hline Oh. & 87 & 2310 & 0,167 & 0,088 & 0,079 & 53,0 & \\
\hline > & 93 & 2165 & 0,179 & 0,085 & 0,094 & 52,6 & \\
\hline$\triangleright$ & 104 & 1990 & 0,133 & 0,093 & 0,040 & 30,1 & \\
\hline , & 117 & 2010 & 0,149 & 0,099 & 0,050 & 33,3 & \\
\hline$\triangleright$ & 130 & 1775 & 0,136 & 0,077 & 0,059 & 43,0 & \\
\hline Er. & 161 & 1755 & 0,181 & 0,102 & 0,079 & 43,7 & $\begin{array}{c}\text { Caseinfett sehrleicht, Filtrat } \\
\text { krystallklar. }\end{array}$ \\
\hline , & 175 & 1715 & 0,187 & 0,089 & 0,098 & 52,6 & do. \\
\hline Ba. & 176 & 3130 & 0,151 & 0,089 & 0,062 & 41,3 & \\
\hline Er. & 178 & 1775 & 0,171 & 0,093 & 0,078 & 45,4 & \\
\hline$\triangleright$ & 180 & 1630 & 0,171 . & 0,096 & 0,075 & 43,7 & \\
\hline Ba. & 203 & 2870 & 0,152 & 0,092 & 0,060 & 39,5 & \\
\hline Sn. & 205 & 1945 & 0,153 & 0,095 & 0,058 & 38,4 & $\begin{array}{l}\text { Ausfällung sehr feinflockig, } \\
\text { Filtrat leicht opalescierend. }\end{array}$ \\
\hline > & 207 & 2200 & 0,180 & 0,095 & $-0,085$ & 46,9 & do. \\
\hline$>$ & 222 & 2375 & 0,148 & 0,099 & 0,049 & 33,3 & » \\
\hline Er. & 223 & 1255 & 0,143 & 0,072 & 0,071 & 49,8 & \\
\hline Mittel & erte & . & 0,184 & 0,105 & 0,079 & 42,9 & \\
\hline
\end{tabular}


Hält man die von anderen Untersuchern gefundenen Caseinmittelwerte bezw. $\mathrm{N}$-Werte daneben, so findet man zum Teil recht erhebliche Abweichungen. So fand Schlossmann (Kalialaunfällung) $61 \% \mathrm{~N}$, Lehmann (Tonfiltration) $1,2 \mathrm{~g}$, Schlossmann (Pukallfilter) $41 \% \mathrm{~N}$, Kobrack (durch Dialysieren) $0,2-0,9 \mathrm{~g}$, Camerer und Söldner(Kali-Alaun) $53,05 \% \mathrm{~N}$ und Zaitscheck (Kali-Alaun) $34,0=40 \% \mathrm{~N}$. Den meinigen ähnliche Werte finden sich also nur bei Schlossmann - $41 \%$ des G. N - und Zaitscheck (Kalialaun) $34-40 \%$ des G. N.

Diese Unstimmigkeiten finden ihre Erklärung in den aufgedeckten natürlichen Schwankungen. Sie sind es auch, welche dazu nötigen, einen Standartwert nur aus einer größeren Untersuchungsreihe abzuleiten.

Ähnliche Schwankungen im Caseingehalte, wie bei der Milch verschiedener Individuen, finden sich aber auch bei der Milch ein und desselben Individuums. So betrug der C. N-Gehalt der Milch von Amme Oh. (5 Untersuchungen) 30-53, der Amme Er. (4 Untersuchungen) 43-52\% des G. N. Rubner und Heubner, ${ }^{1}$ ) die für Stoffwechseluntersuchungen beim Säuglinge die Milch der Mutter auf ihren Caseingehalt nach der $\mathrm{CO}_{2}$-Methode untersuchen ließen, geben C. N-Werte von 29-45\% des G. N an. Derartige Schwankungen scheinen also die Regel zu sein.

Angesichts dieser Eigentümlichkeit ist es eine interessante Aufgabe, die Umstände ausfindig zu machen, mit denen die Schwankungen des Caseingehaltes der Milch bei verschiedenen und denselben Individuen in Verbindung gebracht werden könnten.

Man könnte zunächst daran denken, daß die Dauer der Lactation, die mit der Menge des $\mathrm{G} . \mathrm{N}$ in bestimmten Beziehungen steht, auch hier einen Einfluß ausübe. Um dies zu untersuchen, sind die $\mathrm{N}-\mathrm{Werte}$ der untersuchten Milchen in Tabelle II nach den Lactationstagen geordnet. Man findet in der Tabelle zwar die schon längst bekannte Tatsache, daß der G. N-Gehalt der Frauenmilch vom Beginne bis zum Ende der Lactation eine sinkende Tendenz zeigt, aufs beste bestätigt,

1) Rubner u. Heubner, Zeitschr. f. exp. Path. u. Therap., Bd.I, S. 1. 
doch ein Zusammenhang der Lactationsdauer mit dem Caseingehalt der Milch läßt sichnirgends konstruieren; niedere und hohe Werte finden sich ebenso gut in früheren wie in späten Lactationstagen und zwar sowohl bei verschiedenen, wie bei demselben Individuum; nirgend irgend welche Gesetzmäßigkeit.

Ähnlich liegen die Verhältnisse, wenn man Milchmengen und Caseingehalt in Wechselbeziehung zueinander zu bringen sucht. Es geht aus der Tabelle II hervor, daß es ganz gleichgültig ist, ob viel oder wenig Milch sezerniert wird, die Bildung des Caseins geht ihre eigenen Wege, die offenbar durch ganz andere Faktoren beeinflußt werden.

Auch der Fettgehalt der Milch erwies sich mir ohne jede Bedeutung für den Caseingehalt.

Es ist schwer zu sagen, welche Einflüsse es sind, die das Schwanken des Caseingehaltes der Milch bedingen, ob die wechselnde Zusammensetzung der Nahrung, ob psychische oder nervöse Momente in Frage kommen. Nur soviel läßt sich vermuten, daß man es bei der Sekretion des Caseins mit einer Zellfunktion von besonderer Eigenart zu tun hat. Das glaube ich aus folgenden in Tabelle III niedergelegten Versuchen schließen zu können. Zweck der Versuche war, den Caseingehalt der Tabelle IlI.

\begin{tabular}{|c|c|c|c|c|c|c|c|c|}
\hline \multirow{2}{*}{ Name } & \multirow{2}{*}{\multicolumn{2}{|c|}{$\begin{array}{c}\text { Zeit der } \\
\text { Untersuchung }\end{array}$}} & \multirow{2}{*}{ G. $\mathrm{N}$} & \multirow{2}{*}{ F. N } & \multicolumn{2}{|c|}{$\begin{array}{c}\text { G. } \mathbf{N} \\
\text { in }\end{array}$} & \multicolumn{2}{|c|}{$\begin{array}{c}\text { R. } N \\
\text { in }\end{array}$} \\
\hline & & & & & g & $\begin{array}{c}\% \text { des } \\
\text { G. } \mathrm{N}\end{array}$ & $\mathbf{g}$ & $\begin{array}{c}\% \text { des } \\
\text { G. N }\end{array}$ \\
\hline 1. D. & 19. 1. 5 & Uhr v. & 0,199 & 0,091 & 0,109 & 54,5 & 0,032 & 16,0 \\
\hline & 5 & $>\mathrm{N}$. & 0,189 & 0,092 & 0,097 & 51,2 & 0,031 & 16,5 \\
\hline 3. & 21. I. 5 & N. & 0,175 & 0,087 & 0,088 & 50,3 & 0,033 & 19,0 \\
\hline 4. & 22. I. 5 & $>\quad \mathrm{v}$. & 0,195 & 0,095 & 0,099 & 50,9 & 0,032 & 16,6 \\
\hline 5. & 5 & $=\mathrm{N}$. & 0,181 & 0,095 & 0,086 & 47,3 & 0,038 & 21,2 \\
\hline 6. B. & 23. I. 5 & $>\quad \mathrm{V}$. & 0,140 & 0,075 & 0,065 & 46,6 & 0,025 & 17,6 \\
\hline 7. & 5 & $>\mathrm{N}$. & 0,148 & 0,087 & 0,061 & $\{1,4$ & 0,034 & 23,2 \\
\hline 8. & 24. I. 5 & V. & 0,146 & 0,080 & 0,066 & 45,1 & 0,031 & 21,3 \\
\hline 9. & 5 & N. & 0,145 & 0,094 & 0,051 & 35,0 & 0,032 & 22,4 \\
\hline
\end{tabular}


Milch einzelner Ammen am gleichen Tage je morgens und abends zu bestimmen. Dazu wurden das eine Mal vormittags $5 \mathrm{Uhr}$, das andere Mal nachmittags um $5 \mathrm{Uhr}$ gleiche Mengen entnommen. Neben dem Casein wurde auch der R. N durch Ausfällen sämtlicher Eiweißkörper aus dem caseinfreien Filtrate mit Phosphorwolframsäure bestimmt. Das Ergebnis der Untersuchung war, daß bei den beiden Ammen die Abendmilch sowohl absolut wie prozentual caseinärmer als die Morgenmilch gefunden wurde und zwar bei den ersten Versuchen um 3-5\%, bei den letzten um $10 \%$.

Man wird annehmen können, daß es sich hier gewissermaßen um eine Ermüdungserscheinung der Brustdrüse handelt, zumal auch der R. N, der nach den Untersuchungen von Rietschel ${ }^{1}$ ) zu ungefähr $80 \%$ aus Harnstoff besteht, wie aus der Tabelle III hervorgeht, in der Abendmilch - prozentual in allen 4 Fällen, absolut mit Ausnahme eines Falles - vermehrt ist. Die Zahl der angelegten Kinder und die Häufigkeit der Entleerung spielen dabei wohl eine Rolle. In diesem Falle liegen zwischen Morgen- und Abendmahlzeit noch 2 Stillzeiten dazwischen. Um das Resultat des Versuches noch deutlicher zu gestalten, müßte man die Anfangsmilch der ersten Morgenmilch und die Endmilch der letzten Abendmilch in obigem Sinne untersuchen; dann würden die gefundenen Ausschläge in dem Caseingehalt beider Milchen jedenfalls noch viel deutlicher hervortreten.

Eigentümlich ist nun, wie verschiedenartig und fast gesetzmäßig bei beiden Ammen G. N, F. N und R. N sich zu der konstanten Abnahme des Caseingehaltes der Abendmilch verhalten.

Versuch 1 und 2 bei Amme $D$ zeigt eine Abnahme des Casein-N um 12 und eine Verminderung des G. N um $10 \mathrm{mg}$. Die Gruppe der löslichen Eiweißkörper hat, da der F. N um 1 mg vermehrt, der $R$. $N$ um $1 \mathrm{mg}$ vermindert ist, eine Zunahme von $2 \mathrm{mg}$ erfahren.

Im Versuch 4 und 5 beträgt die C. N-Differenz 13, die des G. N $14 \mathrm{mg}$, während der F. N bei einer Zunahme des R. N um $6 \mathrm{mg}$ auf Kosten der löslichen Eiweißkörper nahezu derselbe

1) Rietschel, Jahrb. f. Kinderheilk., Bd. 64, S. 125. 
geblieben ist. Beide Versuche haben das Gemeinsame, daß die Differenz des $\mathrm{C}$. $\mathrm{N}$ sich fast mit der Abnahme des $\mathrm{G}$. $\mathrm{N}$ deckt. Das würde heißen: die verminderte Arbeitsleistung der Brustdrüsenzellen infolge der Ermüdung äußert sich in diesem Falle so, daß die aus dem Blutserum zum Aufbau des Caseinmoleküls den Zellen zugeführten Eiweißstoffe von vornherein nur in beschränktem Maße verarbeitet .werden.

Ganz anders liegen die Verhältnisse bei Amme B. Im Versuch 6 und 7 steht der Abnahme des C. $\mathrm{N}$ der Abendmilch um $4 \mathrm{mg}$ eine Zunahme des $\mathrm{F}$. $\mathrm{N}$ um $12 \mathrm{mg}$ gegenüber. In diesen $12 \mathrm{mg}$ sind enthalten das Plus von $8 \mathrm{mg}$ des $\mathrm{G}$. $\mathrm{N}$, die anscheinend dem $R$. N (十 $9 \mathrm{mg}$ ) zugute kommen; es müssen demnach die noch übrig bleibenden $4 \mathrm{mg} \mathrm{N}$, die auf die Gruppe der löslichen Eiweißkörper entfallen, der Differenz von $4 \mathrm{mg}$ des C. $\mathrm{N}$ analog sein.

Ähnlich, doch viel prägnanter ist das Resultat des Versuches 8 und 9. Der Abnahme des C. N um $15 \mathrm{mg}$ entspricht eine Zunahme des F. N um $14 \mathrm{mg}$, während G. $\mathrm{N}$ und R. N nur eine geringe Veränderung um $1 \mathrm{mg}$ im negativen und positiven Sinne zeigen. Das heißt, die $15 \mathrm{mg} \mathrm{C}$. N, welche die Abendmilch weniger enthält als die Morgenmilch, sind fast vollständig als den löslichen Eiweißkörpern angehöriger $\mathrm{N}$ in den F. $\mathbf{N}$ übergegangen. Demnach würde sich bei Amme B. die Minderfunktion der Brustdrüse infolge Ermüdung so äußern, daß diejenigen $\mathrm{N}$-haltigen Stoffe, aus denen der Aufbau des Caseinmoleküls erfolgt, zwar (im Gegensatz zu Amme D) in derselben Menge von den Drüsenzellen aufgenommen werden, aber ohne zu Casein verarbeitet worden zu sein, die Zelle passieren und in minder spezifischer Form in der Milch erscheinen.

Ist die Theorie der Ermüdung für die Abnahme des Caseingehaltes der Milch in den Abendstunden richtig, so muß umgekehrt der Caseingehalt der Morgenmilch, da die Brustdrüse über Nacht außer Funktion war, höher als der Caseingehalt der Abendmilch am vorhergehenden Tage, dagegen der $R . N$ der Morgenmilch im Vergleich zu dem der Abendmilch vermindert sein. Das wird man bestätigt finden, wenn man die Versuche 3 u. 4 und 7 u. 8 miteinander vergleicht. Versuch 4 
zeigt gegenüber 3 eine Zunahme des G. N um $20 \mathrm{mg}$. Demnach haben neben dem $\mathrm{C}$. $\mathrm{N}$, da der $\mathrm{R}$. $\mathrm{N}$ um $1 \mathrm{mg}$ abgenommen hat, auch die löslichen Eiweißkörper eine Vermehrung erfahren und zwar um rund $9 \mathrm{mg}$. Im Versuch 8 weist der C. $\mathrm{N}$ gegenüber 8 eine Zunahme um $5 \mathrm{mg}$ auf bei gleichzeitigem Sinken des G. $\mathrm{N}$ um 2, des F. N um 7,0 mg.

Beide Doppelversuche illustrieren somit wieder in interessanter Weise die Verschiedenheit des Sekretionsmechanismus beider Ammen. Bei Amme D wird das Plus an C. $N$ erzielt durch Herbeischaffung neuer $\mathrm{N}$-haltiger Substanz (Zunahme des G. $\mathrm{N}$ um $20 \mathrm{mg}$ ), während bei Amme $\mathrm{B}$, bei der der G. $\mathrm{N}$ ziemlich auf dieselbe Höhe eingestellt zu sein scheint, die Vermehrung des $\mathrm{C}$. $\mathrm{N}$ auf Kosten der löslichen Eiweißkörper erfolgt.

Warum die sekretorischen Vorgänge in den Brustdrüsenzellen beider Ammen sich so verschiedenartig gestalten, läßt sich auf Grund dieser wenigen Versuche kaum sagen. Amme D befand sich zur Zeit der Untersuchung etwa am 66. Lactationstage und hatte eine Tagesmilchmenge von $2000 \mathrm{ccm}$, Amme B hatte etwa 256 Stilltage hinter sich und lieferte zur Zeit der Untersuchung im Mittel $2700 \mathrm{ccm}$ Milch. Es hat hiernach den Anschein, als ob Lactationstermin und Milchmenge von einigem Einfluß in dieser Frage sein könnten.

Nach den Ergebnissen der in Tabelle III niedergelegten Versuche könnte man daran denken, auch bei der Anfangsund Endmilch der Brustdrüse ähnliche Eigentümlichkeiten der Sekretion zu finden. Das hat sich jedoch nicht bestätigt. In zwei Probeversuchen, in denen Milch einer Amme verwertet warde, konnte zwar eine Verminderung des G. $\mathbf{N}$ der Endmilch festgestellt werden, ohne andere gesetzmäßige Verschiebungen. Ich lasse beide Versuche hier folgen (Tabelle IV), ohne ihren Resultaten einen besonderen Wert beizumessen.

Dem Casein als dem spezifischen Nucleoprotein der Milch hat man eine andere Gruppe von Eiweißkörpern gegenübergestellt, die man im Gegensatz zu dem Casein nicht ganz korrekt als die Gruppe der löslichen Eiweißkörper bezeichnet. Dahin gehören das Albumin, Globulin und «Opalisin».

Um die Menge des auf sie in ihrer Gesamtheit entfal- 
lenden $\mathrm{N}$ genau bestimmen $\mathrm{zu}$ können, muß man die Menge des R. N von dem F. N abziehen, genau so, wie man auch das Gesamteiweiß durch Subtraktion des R. N vom G. $N$ erhältt.

Tabelle IV.

\begin{tabular}{|c|c|c|c|c|c|}
\hline & \multirow{2}{*}{ G. $\mathrm{N}$} & \multirow{2}{*}{ F. N } & \multicolumn{2}{|c|}{ C. $\mathrm{N}$} & \multirow{2}{*}{$\begin{array}{c}\text { R. N } \\
\%\end{array}$} \\
\hline & & & g & $\%$ & \\
\hline A. $\left.{ }^{*}\right)$ & 0,182 & 0,090 & 0,092 & 50,65 & 20,0 \\
\hline E. **) & 0,178 & 0,087 & 0,091 & 51,34 & 20,0 \\
\hline A. & 0,186 & 0,093 & 0,093 & 50,0 & 21,87 \\
\hline E. & 0,182 & 0,084 & 0,098 & 53,8 & 21,97 \\
\hline
\end{tabular}

*) $\mathbf{A}=$ Anfangsmilch.

**) $\mathrm{E}=$ Endmilch.

Nach Camerer und Söldner ${ }^{1}$ ) schwankt der R. N zwischen 12 und $21 \%$ des G. N; Rietschel fand ähnliche Werte. Meine R. N-Analysen, deren Resultate in Tabelle $\mathbf{V}$ enthalten sind, lassen R. N-Werte unter $16 \%$ überhaupt nicht erkennen, vielmehr bewegen sie sich fast durchweg mehr in der Nähe von $20 \%$, sind also ziemlich hoch. Die Konstanz der hohen R.N-Werte ist auffallend. Unvollkommene Ausfällung der Eiweißkörper kann nicht vorliegen. Die Abscheidung derselben wurde so ausgeführt, daß zu $100 \mathrm{ccm}$ des klaren (caseinfreien) Filtrates solange Phosphorwolframsäure zugesetzt wurde, bis keine Fällung mehr eintrat. Im klaren Filtrat wurde dann der $\mathrm{N}$ nach Kjeldahl bestimmt. Die einzige denkbare Erklärung für die durchschnittlich niedrigeren $\mathrm{R}$. NWerte der Autoren ist die, daß durch die Totalfällung der Eiweißkörper, wie sie z. B. von Camerer und Söldner mit Alménscher Lösung vorgenommen wurde, Teile der Restsubstanzen mitgerissen werden, was bei einer mehr fraktionierten Fällung (erst Casein, dann lösliche Eiweißkörper) schwerer möglich ist. Auch die Verschiedenartigkeit der Fällungsmittel kann nicht in Frage kommen, da bei einem Kontrollversuche an derselben Milch der nach zwei Fällungsarten gewonnene R. $\mathrm{N}$ in der gleichen Menge vorhanden war. Der Durch-

1) Camerer u. Söldner, Zeitschr. f. Biol., Bd. XV, S. 535. 
schnittswert des $\mathrm{R}$. $\mathrm{N}$ meiner Untersuchungen beträgt also rund 20\% des G. N. Dieser hohe R. N der Frauenmilch verdient eine besondere Berücksichtigung. Da er sich nämlich, wie schon hervorgehoben, zu rund $80 \%$ aus Harnstoff und aufierdem aus anderen Eiweißabbauprodukten zusammensetzt, so spielen also rund $1 / 5$ des G. $N$ als Nahrungsmittel gar keine oder nur eine untergeordnete Rolle.

Tabelle V.

\begin{tabular}{|c|c|c|c|c|c|c|c|c|c|}
\hline & \multirow{2}{*}{ G. N } & \multirow{2}{*}{$\mathrm{N}$} & \multicolumn{2}{|c|}{ R. $\mathrm{N}$} & \multirow{2}{*}{$\left|\begin{array}{c}\text { G.N }- \text { R.N } \\
=\text { Ges. Eiw. } \\
\mathrm{N} \\
\text { (g Eiweiß) }\end{array}\right|$} & \multicolumn{2}{|c|}{$\begin{array}{l}\text { C. } \mathbf{N} \\
\text { in }\end{array}$} & \multicolumn{2}{|c|}{$\begin{array}{l}\text { F. } N-R \cdot N=N \\
\text { des lösl. Eiw. }\end{array}$} \\
\hline & & & g & $\begin{array}{c}\% \\
\text { G. N }\end{array}$ & & g & $\begin{array}{l}\% \text { des } \\
\text { Ges. } \\
\text { Eiw. N }\end{array}$ & $\mathbf{g}$ & $\begin{array}{l}\% \text { des } \\
\text { Ges. } \\
\text { Eiw. N }\end{array}$ \\
\hline Misc & 40 & 0 & 5 & 17,5 & $\begin{array}{c}0,115 \\
(0,72)\end{array}$ & 0,066 & 57,4 & 0,049 & 42,6 \\
\hline Anme B. & 0,145 & 0,083 & 0,030 & 20,7 & $\begin{array}{c}0,115 \\
(0,72)\end{array}$ & 0,062 & 54,0 & 0,053 & 46,0 \\
\hline Mischmilch & 0,151 & 0,087 & 0,029 & 19,5 & $\begin{array}{c}0,122 \\
(0,76)\end{array}$ & 0,064 & 52,5 & 0,058 & 47,5 \\
\hline$\triangleright$ & 0,157 & 0,084 & 0,034 & 21,9 & $\begin{array}{c}0,123 \\
(0,77)\end{array}$ & 0,073 & 59,4 & 0,050 & 40,6 \\
\hline > & 0,176 & 0,098 & 0,036 & 20,6 & $\begin{array}{r}0,140 \\
(0,87)\end{array}$ & 0,078 & 55,7 & 0,062 & 44,3 \\
\hline Anme $\mathrm{E}$. & 0,182 & 0,090 & 0,036 & 20,0 & $\begin{array}{c}0,146 \\
(0,91)\end{array}$ & 0,090 & 63,0 & 0,054 & 37,0 \\
\hline Mischmilch & & 0,114 & 0,039 & 20,9 & $\begin{array}{l}0,146 \\
(0,91)\end{array}$ & 0,071 & 48,7 & 0,075 & 51,3 \\
\hline Anme D. & & 0,092 & 0,033 & 17,5 & $\begin{array}{l}0,155 \\
(0,97)\end{array}$ & 0,096 & 62,0 & 0,059 & 38,0 \\
\hline Mischmilch & 0,194 & 0,103 & 0,039 & 19,9 & $\begin{array}{c}0,155 \\
(0,97)\end{array}$ & 0,091 & 58,7 & 0,064 & 41,3 \\
\hline 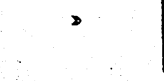 & 0,244 & 0,111 & 0,042 & 17,1 & $\begin{array}{r}0,202 \\
(1,26)\end{array}$ & 0,133 & 65,9 & 0,069 & 34,1 \\
\hline
\end{tabular}

Die Verteilung der Eiweißkörper in diesen 10 untersuchten Milchen würde nun nach der Tabelle $\mathrm{V}$ folgende sein: Der Gesamteiweißgehalt, dessen Höhe 0,72-1,26g auf $100 \mathrm{ccm}$ Milch beträgt, besteht zu 48,7-65,9\% - Mittel 57,7\% aus Casein und zu 38,0-51,3\% - Mittel 42,3\% - aus lösiichen Eiweißkörpern. Standartwerte sind das jedoch nicht. 
Schon aus Tabelle II kann man andere, etwas niedrigere Zahlen für das Casein erhalten.

Vergleicht man die Mengenverhältnisse von Casein und löslichen Eiweißstoffen der für Säuglingsernährung in Betracht kommenden Tiermilchen mit der menschlichen Milch, so ergibt sich folgendes:
Kuhmilch $10: 1$
Eselinnenmilch 2,4 : 1
berechnet nach Raudnitz, Handbuch der Kinderheilkunde, v. Pfaundler-Schlossmann,

Frauenmilch 1,3:1 (Mittel aus 10 Untersuchungen).

Es ist also ersichtlich, wie schon lange bekannt, daß die Eselinnenmilch in bezug auf die Eiweißverteilung der Frauenmilch am ähnlichsten ist.

Dieser hohe Gehalt an löslichen Eiweißkörpern, der an sich schon für die Verdauung der Frauenmilch gewisse Eigenarten bedingt, erfährt noch durch die Labgerinnung im Magen eine nicht unbeträchtliche Vermehrung. Noch bis in die jüngste Zeit hielt man eine Labfällung der Frauenmilch im Reagenzglase für nicht ausführbar und sah sogar darin einen der Hauptunterschiede zwischen Kuh- und Frauenmilch. Erst durch die neueren Untersuchungen von Fuld und Wohlgemuth (l. c.), Kreidl und Neumann ${ }^{1}$ ) und von Engel (l. c.) wurde die Möglichkeit einer Labung auch für die Frauenmilch festgestellt. Es ist nicht meine Absicht, hier den Vorgang der Labfällung als solchen einer Erörterung zu unterziehen - ich verweise da auf die betreffenden Arbeiten (Literatur bei Schlossmann und Engelª)) - . Nur auf die dabei sich vollziehende Verschiebung in der Stickstoffverteilung möchte ich an der Hand einiger Versuche etwas näher eingehen. Sie pflegt sich in der Weise abzuspielen, daß von dem Caseinmolekül ein Atomkomplex abgesprengt wird, der als lösliches Eiweiß oder eine dem Eiweiß nahestehende Substanz in die Molke übergeht. Die Menge derselben, welche sich durch Subtraktion des Essigsäurefiltrat-N vom Labfiltrat-N ergibt, ist bei der Frauenmilch eine ziemlich

1) Kreidl und Neumann, Pflügers Arch., Bd. CXXIII, S. 523.

2) Schlossmannu. Engel, «Milchgerinnung» in 0ppenheimers Handbuch d. Biochem., Bd. III, Jena 1909, b. G. Fischer. 
erhebliche und beträgt nach den Untersuchungen von Engel ${ }^{1}$ ) und Friedheim ${ }^{2}$ ) bei den meisten Milchen annähernd $10 \%$ des G. N. Meine in Tabelle 6 niedergelegten und nach der bei Friedheim beschriebenen Methode ausgeführten Analysen bestätigen im wesentlichen die Engel-Friedheimschen Untersuchungsresultate: Die Zunahme des Molken- $N$ bewegt sich in den meisten Fällen um 10\% des G. $N$ herum, doch kommen auch Werte von 6 und 5\% vor. Ein wesentlicher Einflu $B$ der Dauer der Labwirkung auf die Größe der Eiweißabspaltung lie $B$ sich nicht konstatieren.

Tabelle VI.

\begin{tabular}{|c|c|c|c|c|c|c|}
\hline \multirow[t]{2}{*}{ G. $\mathrm{N}$} & \multicolumn{2}{|c|}{$\begin{array}{l}\text { E. F. } N^{*} \text { ) } \\
\text { in }\end{array}$} & \multicolumn{2}{|c|}{$\begin{array}{c}\text { L. F. } \mathrm{N}^{* *} \text { ) } \\
\text { in }\end{array}$} & \multicolumn{2}{|c|}{ L. F. ${ }_{\text {in }}^{\mathrm{N}}-\mathrm{E} \cdot \mathrm{F} \cdot \mathrm{N}$} \\
\hline & g & $\%$ & g & $\%$ & g & $\%$ \\
\hline 0,140 & 0,074 & 53,2 & 0,091 & 64,0 & 0,016 & 10,8 \\
\hline 0,151 & 0,087 & 57,4 & 0,095 & 62,6 & 0,008 & 5,1 \\
\hline 0,156 & 0,080 & 51,1 & 0,096 & 61,3 & 0,016 & 10,2 \\
\hline 0,157 & 0,084 & 53,8 & 0,095 & 60,5 & 0,010 & 6,7 \\
\hline 0,176 & 0,098 & 55,5 & 0,116 & 65,9 & 0,018 & 10,5 \\
\hline 0,185 & 0,114 & 61,5 & 0,128 & 69,1 & 0,014 & 7,6 \\
\hline 0,194 & 0,103 & 53,2 & 0,122 & 62,6 & 0,018 & 9,4 \\
\hline 0,244 & 0,111 & 45,5 & 0,139 & 57,1 & 0,018 & 11,6 \\
\hline
\end{tabular}

*) E. F. $\mathrm{N}=\mathrm{N}$-Gehalt der Essigsäuremolke.

**) L.F. $\mathrm{N}=$, , Labmolke.

Der letzte Teil dieser Arbeit beschäftigt sich mit einigen Fragen, die sich auf Art und Verteilung der Eiweißkörper in der Molke beziehen, ein Unternehmen, das jedoch wegen der Schwierigkeit des zu behandelnden Stoffes zu keinem bindenden Abschlusse gelangen konnte.

Gelegentlich einer Bestimmung des $\mathrm{R}$. $\mathrm{N}$ mit Hilfe von Phosphorwolframsäure wurde gleichzeitig versucht, ob man nicht durch einfaches Aufkochen der Essigsäuremolke zu den-

1) Engel, Münch. med. Wochenschr., 1910.

2) Friedheim, Bioch. Zeitschr., Bd. XIX, S. 132. 
selben R. N-Werten gelangen könnte, wie sie jene Methode ergibt. Das war nun keineswegs der Fall, weder bei diesem noch bei allen folgenden Versuchen. Vielmehr überstiegen die durch Kochen der Molken gewonnenen R. N-Werte, wenn man sie so nennen darf, den eigentlichen $R$. $N$ um ein Bedeutendes.

Die Ursache davon kann zweierlei sein: Entweder handelt es sich um eine unvollkommene Ausfällung der zur Gruppe der Albumine und Globuline gehörigen Eiweißkörper, oder die Molke enthält noch andere N-haltige Stoffe, die durch den Kochprozeß nicht koaguliert werden.

Eine quantitative Fällung des Albumins und Globulins durch einfaches Kochen ist im allgemeinen nicht ohne weiteres möglich. Während es bei einer stark verdünnten Hühnereiweißlösung leicht gelingt, das Eiweiß quantitativ durch kurzdauerndes Aufkochen zu fällen, sodaß man ein absolut klares, nicht mehr auf Phosphorwolframsäure reagierendes Filtrat erhält, stößt man bei der Fällung des Albumins und Globulins aus der Molke auf Schwierigkeiten. Kocht man dieselbe ein paar Sekunden, so findet allerdings eine Konzentration des Eiweißes in Form schon makroskopisch sichtbarer Flocken statt, aber das klare Filtrat trübt sich von neuem, wenn man es noch einmal kocht. Setzt man jedoch der Molke eine kleine Menge eines neutralen Salzes (etwa $\mathrm{NaCl}$ ) zu, so genügt ein paarmaliges Aufkochen, um die Ausfällung quantitativ zu machen, - das klare Filtrat gibt alsdann beim Kochen keine neue Trübung. Dasselbe kann man ohne Anwendung eines Salzes erreichen, wenn man längere Zeit etwa 1/2 Stunde lang kocht, wovon ich mich durch vergleichende $\mathrm{N}$-Bestimmungen bei gleichem Ausgangsmateriale überzeugt habe.

Im allgemeinen wurden die Versuche so ausgeführt, daß die Molke nach Zusatz einer Messerspitze NaCl 5 Minuten im Sieden erhalten, dann aufs Volumen ergänzt und filtriert wurde. Im klaren Filtrate wurde der $\mathrm{N}$-Gehalt nach Kjeldahl bestimmt. Die so erhaltenen Zahlen, welche auf $100 \mathrm{ccm}$ Milch umgerechnet wurden, zeigt Tabelle VII, Spalte 5a. Sie übersteigen, wie man sieht, die entsprechenden R. N-Zahlen, die aus Tabelle VIII zu ersehen sind, um ein Bedeutendes, im Durch- 
schnitt um ungefähr $20 \mathrm{mg}$, die also auf Eiweißkörper von anderem Charakter zu beziehen wären.

Ähnlich wie der Einwirkung der Siedehitze gegenüber verhält sich die Molke, resp. deren Eiweißkörper auf Zusatz von Alkohol. Um dessen Einwirkung möglichst intensiv zu gestalten, wurden zu 100 Teilen Molke 200 Teile $96 \%$ igen Alkohols hinzugesetzt und die Mischung verschlossen solange stehen gelassen, bis eine Koagulation in Form von makroskopisch sichtbaren Flocken eingetreten war; das ging gewöhnlich schneller nach vorheriger Neutralisation, wodurch jedoch die Größe der Fällung, wie ein Probeversuch ergab, nicht beeinflußt wurde. Das klare Filtrat, welches in allen Fällen mit Alkohol aufs Volumen ergänzt wurde, zeigte, wenn auch mehrere Stunden stehen gelassen, keine neue Trübung, was für eine quantitative Fällung spricht. Der $\mathrm{N}$-Gehalt desselben, in 5 Versuchen bestimmt (Tabelle VII, Spalte 7 a), nähert sich den entsprechenden Werten des Kochfiltrates, stimmt jedoch nicht völlig mit denselben überein.

Endlich und nicht am wenigsten spricht auch die Trübung, welche nach Sättigung der Molke mit NaCl in ziemlicher Stärke auftritt, entschieden für das Vorhandensein noch anderer Eiweißkörper, da Albumin und Globulin in gesättigten $\mathrm{NaCl-Lösungen}$ nicht verändert werden. Daher muß es interessant sein, festzustellen, in wieweit die Resultate der Koch- und Alkoholfällung einerseits mit der $\mathrm{NaCl}-\mathrm{Fällung}$, anderseits miteinander in Beziehung gebracht werden könnten. (2 Versuche, die den NGehalt im Filtrate der NaCl-Fällung bestimmen sollten, waren leider so ungenau ausgefallen, daß sie nicht zu gebrauchen waren.) Was die Art des oder der fraglichen N-haltigen Körper angeht, so handelt es sich entweder um genuine, d. h. schon in der frischen Milch vorhandene Stoffe, oder aber um Kunstprodukte, die durch Einwirkung der betreffenden Agentien auf die Eiweißstoffe - Absprengung N-haltiger Atomkomplexe vom Caseinmolekül oder Umwandlung der löslichen Eiweißtoffe - zustande kommen. So könnten sich Albumosen und Peptone und andere Körper bilden.

Um dàrüber Klarheit zu erhalten, wurde die nach NaCl- 
A. Frehn,

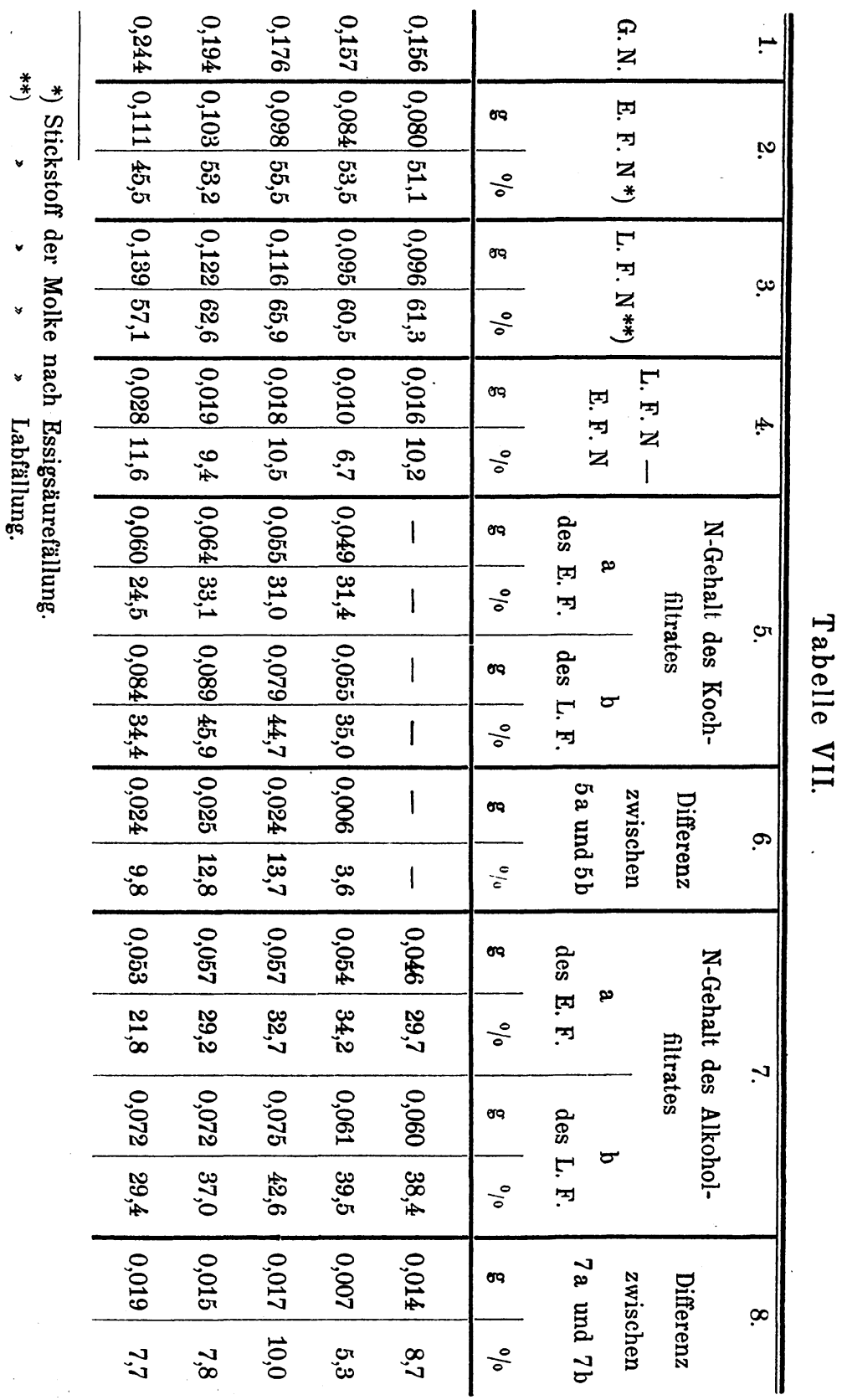


Zusatz durch Kochen von Albumin und Globulin befreite Molke zunächst auf ihr Verhalten den verschiedenen Eiweißreagentien gegenüber untersucht. Das Resultat war folgendes:

1. Biuretprobe ziemlich stark +

2. Xanthoproteinprobe +

3. Millonsche Reaktion: Gelbfärbung, keine Purpurfärbung.

4. Schwefelbleiprobe +

5. Zusatz gleicher Volumina gesättigter NaCl-Lösung und Essigsäure, keine Fällung.

6. Auf Zusatz von konzentrierter $\mathrm{HNO}_{3}$ ganz schwache Opalescenz, die beim Erwärmen verschwindet; bei stärkerem Erwärmen schwache Gelbfärbung.

7. Auf Zusatz von $\mathrm{CuSO}_{4}$ keine Fällung.

8. Essigsäure + Ferrocyankalium - kein Niederschlag.

9. Phosphorwolframsäure $=$ mäßig starke Trübung.

10. Sättigung mit $\mathrm{NaCl}$ deutlich hervortretende Trübung.

Zum Vergleiche müssen obige Proben ebenfalls an der durch Alkohol vom Albumin und Globulin befreiten Molke angestellt werden. Weitgehende Schlüsse werden auch dann nicht aus den Resultaten zu ziehen sein. Auffallend ist immerhin, daß die als Albumosenreaktionen in den Lehrbüchern (HoppeSeyler, Röhmann) ${ }^{1}$ ) angegebenen Proben (5-8), vor allem die Essigsäureferrocyankaliumprobe, die zur Kontrolle mehrere Male ausgeführt wurden, negativ waren. Demnach scheinen Albumosen in nachweisbarer Menge nicht vorhanden zu sein, obwohl ihre Bildung aus dem Albumin bei dem einige Minuten auf die saure Molke einwirkenden Kochprozesse immerhin theoretisch möglich wäre.

Es spricht aber auch der folgende Versuch gegen das Vorhandensein von Albumosen, die sich durch Einwirkung der Siedehitze aus dem Eiweiß gebildet haben könnten. Es wurde dieselbe Molke, das eine Mal, wie gewöhnlich, nur etwa 5 Minuten, das andere Mal 1/2 Stunde gekocht. Die im klaren Filtrate darauf vorgenommene N-Bestimmung ergab beide Male fast

1) Lehrbücher. 
dieselben Werte, 0,089 und 0,090 (auf $100 \mathrm{~g}$ Milch bezogen). Würden überhaupt durch das Kochen in diesem Falle Albumosen gebildet werden, so wäre zu erwarten, daß durch eine längere Dauer desselben auch eine größere Menge gebildet würde.

Ebensowenig könnten die fraglichen Eiweißstoffe des Kochund Alkoholfiltrates in Lösung gebliebene Acidalbumine sein, denn diese werden ebenso wie ihre Muttersubstanzen durch die Siedehitze bei Anwesenheit einer gewissen Menge von Neutralsalz gefällt. Ähnlich wird ihr Verhalten gegenüber der Einwirkung des Alkohols sein, wenigstens ließ ein mit derselben Molke angestellter Doppelversuch, wobei jene das eine Mal neutralisiert wurde, das andere Mal unverändert blieb, keinerlei Unterschied in den N-Zahlen erkennen.

Somit bleiben für die Herkunft der fraglichen Eiweißstoffe nur 2 Möglichkeiten übrig, entweder sie stammen vom Casein, oder es sind native Eiweißstoffe der Milch. Daß durch die Einwirkung der Essigsäure allein vom Casein-Molekül ein N-haltiger Atomenkomplex abgesprengt wird, ist bei der labilen Struktur des Caseins denkbar, doch bei der starken Verdünnung der Säure nicht wahrscheinlich. Der Beweis dafür kann allerdings vorläufig nicht erbracht werden.

Näher liegt es, in dem betreffenden $\mathrm{N}$-haltigen Stoffe einen in die Gruppe der löslichen Eiweißstoffe gehörigen Körper anzunehmen, der vielleicht mit dem Opalisin Wroblewskis identisch sein könnte. Diese Vermutung ist deshalb berechtigt, weil das Opalisin gewonnen wurde aus den Mutterlaugen der Essigsäurefällung des Caseins durch Aussalzen mit $\mathrm{NaCl}$, ebenso wie auch der hier in Frage kommende Eiweißstoff aus dem Kochfiltrat der Molke durch Sättigung mit NaCl gefällt wurde. $\mathrm{Ob}$ die Annahme richtig ist, das festzustellen muß weiteren Versuchen anheimgegeben werden.

Um nun bei den etwas von einander abweichenden Ergebnissen der Koch- und Alkoholfällung der Molke wenigstens die Mengenverhältnisse der löslichen Eiweißkörper genauer angeben zu können, wurde aus den in Tabelle VII (Spalte 5a und 7a) enthaltenen N-Zahlen des Koch- und Alkoholfiltrates das Mittel in Rechnung gezogen (Tab. VIII). Der auf Albumin 
und Globulin entfallende $\mathrm{N}$ würde sich dann ergeben aus der Differenz zwischen dem N-Gehalt der Essigsäuremolken und obigen N-Mittelwerten. Der dem unbekannten Eiweißkörper angehörige $\mathrm{N}$ aus der Differenz zwischen $\mathrm{N}$-Mittelwerten und dem R. N. Tabelle VIII enthält die so gefundenen Werte. Die untersuchten Milchsorten mit einem G. $\mathrm{N}$ von $0,157-0,244 \mathrm{~g}$ enthalten an Albumin- und Globulinstickstoff $0,033-0,054 \mathrm{~g}$ (in Prozent des G. $\mathrm{N}$ ziemlich konstanterweise $21-23$ ), an $\mathrm{N}$, der auf den unbekannten Eiweißkörper entfällt, 0,015-0,022 g $(6-11 \%$ des G. N). Zieht man das Mittel aus allen vier Versuchen und rechnet auf Eiweiß um, so ergibt sich folgende Eiweißverteilung: Eine Milch mit einem Eiweißgehalt von 1,0 g würde enthalten $0,6 \mathrm{~g}$ Casein, $0,3 \mathrm{~g}$ Albumin + Globulin und $0,1 \mathrm{~g}$ des unbekannten Eiweißstoffes.

Tabelle VIII.

\begin{tabular}{|c|c|c|c|c|c|c|c|c|c|c|}
\hline \multirow{2}{*}{$\begin{array}{c}\text { G. N } \\
\mathrm{g}\end{array}$} & \multicolumn{2}{|c|}{ E. F. N } & \multicolumn{2}{|c|}{ R. N } & \multicolumn{2}{|c|}{$\begin{array}{c}\text { N-Mittelwerte } \\
\text { aus Alkohol- } \\
\text { u. Kochfiltrat } \\
\text { des E. F. }\end{array}$} & \multicolumn{2}{|c|}{$\begin{aligned} & \text { E. F. N } \\
& \text { - } \text { N-Mittel- } \\
& \text { werte } \\
&= \text { Albumin } \\
&+ \text { Globulin }\end{aligned}$} & \multicolumn{2}{|c|}{$\begin{array}{c}\text { N-Mittelwerte } \\
\text { - Rest-N } \\
=\text { unbekann- } \\
\text { ter Eiweiß- } \\
\text { körper }\end{array}$} \\
\hline & g & $\%$ & g & $\%$ & $\mathrm{~g}$ & $\%$ & g & $\%$ & g & $0 / 0$ \\
\hline 0,157 & 0,084 & 53,8 & 0,034 & 21,9 & 0,051 & 32,7 & 0,033 & 21,0 & 0,017 & 10,8 \\
\hline 0,176 & 0,098 & 55,5 & 0,036 & 20,7 & 0,056 & 31,8 & 0,042 & 23,6 & 0,020 & 11,2 \\
\hline 0,194 & 0,103 & 53,2 & 0,039 & 20,0 & 0,061 & 31,2 & 0,042 & 22,0 & 0,022 & 11,2 \\
\hline 0,244 & 0,111 & 45,5 & 0,042 & 17,1 & 0,057 & 23,1 & 0,054 & 22,3 & 0,015 & 6,0 \\
\hline
\end{tabular}

Wie die Essigsäuremolke wurde auch die Labmolke der Einwirkung des Kochens und des Alkohols unterzogen, um zu sehen, ob und inwieweit der bei der Labung in die Molken übergegangene lösliche Eiweißkörper mitgefällt wird, was wieder einen Schluß auf die Art dieses Eiweißkörpers zuläßt. Das wird sich nachweisen lassen, wenn man die Differenzen aus dem Kochfiltrat der Essigsäure- und Labmolke mit der Menge des bei der Labung abgespaltenen Eiweißstoffes vergleicht. Die Ergebnisse der. Untersuchungen enthält Tabelle VII. Die Differenz zwischen beiden Kochfiltraten (Spalte 6) 
ist in 2 Fällen kleiner, in 2 Fällen größer, als die N-Zahlen des Labeiweißes (Spalte 4). Das Plus an $\mathrm{N}$ in den beiden Fällen kann darauf zurückzuführen sein, daß entweder von der Essigsäuremolke zu viel, oder von der Labmolke zu wenig gefällt wurde, was natürlich nicht mehr entschieden werden kann. Die Differenz zwischen beiden Alkoholfiltraten (Spalte 8) ist durchweg nur um ein paar Milligramm niedriger als die $\mathrm{N}-$ Zahlen in Spalte 4. Aus alledem ist die Folgerung berechtigt, daß der bei der Labung in die Molke übergegangene lösliche Eiweißkörper durch Einwirkung von Siedehitze und Alkohol in der angewandten Konzentration nur zu einem kleinen Teile koaguliert wird.

Die Arbeit wurde auf Veranlassung und unter Leitung von Dr. Engel, Düsseldorf, ausgeführt.

\title{
Berichtigung betreffend «Londons Polyfistelmethode».
}

\author{
Von \\ W. W. Sawitsch,
}

Assistent am physiologischen Laboratorium der Kaiserlich Militärmedizinischen Akademie in St. Petersburg.

(Der Redaktion zugegangen am 24. Februar 1910.)

Im «Handbuch der Biochemischen Arbeitsmethoden», herausgegeben von Prof. Abderhalden, unterzieht London die Grundlagen der operativen Methodik zur Untersuchung der Verdauungsprozesse einer genauen Untersuchung. Dabei führt er in der historischen Entwickelung dieser Frage an: 1. «das Prinzip der operativen Isolierung der sezernierenden Oberfläche», welches von Thiry und Heidenhain vorgeschlagen worden ist und 2. das von Pawlow vorgeschlagene neue Prinzip, und zwar «die operative Isolierung eines Teils des Ganzen unter Erhaltung der sekretorisch nervösen Verbindung zwischen den einzelnen Teilen`(S. 83, Bd. III); indessen soll «die stetige Abfuhr der Säfte nach außen im Verdauungstraktus und zugleich auch im Gesamtorganismus abnorme Bedingungen schaffen». Es ist möglich, daß die Ausschaltung der Produkte der Drüsentätigkeit solche Verhältnisse schafft, die den pathologischen sehr nahe sind. 